\title{
Evaluation of Child Perceptions Questionnaire on Oral Health Related Quality of Life among School Children with Various Orofacial Conditions in Mysore, India
}

\author{
Sushma Rudraswamy ${ }^{1 *}$, Vijai S. ${ }^{2}$, Nagabhushana Doggalli ${ }^{3}$, Maurya Manjunath ${ }^{4} \&$ Sunitha Siddana $^{5}$ \\ ${ }^{I}$ Department of Public Health Dentistry, J.S.S Dental College and Hospital, J.S.S Academy of Higher Education and Research, Mysore, India \\ ${ }^{2}$ Department of Pedodontics, Malabar Dental College \& Research Centre, Malapuram, Kerala, India. \\ ${ }^{3}$ Department of Oral Medicine and Radiology, J.S.S Dental College and Hospital, J.S.S Academy of Higher Education and Research, Mysore, India. \\ ${ }^{4}$ Department of Public Health Dentistry, J.S.S Dental College and Hospital, J.S.S Academy of Higher Education and Research, Mysore, India. \\ ${ }^{5}$ Department of Public Health Dentistry, J.S.S Dental College and Hospital, J.S.S Academy of Higher Education and Research, Mysore, India. \\ *Corresponding Author Email: sushmarudraswamy@gmail.com
}

DOI: $10.38177 / A J B S R .2020 .2302$

\section{ABSTRACT}

Objective: To know the reliability and validity of child perceptions questionnaire among 11-14 years old children.

Methodology: The clinical data on caries status, malocclusion, dental opacities and gingivitis were collected among 90 school children aged 11-14 years who also completed the child perceptions questionnaire that included 37 items enquiring about impacts on four health domains (subscales).

Results: An acceptable internal consistency with the Cronbach's alpha for the total scale was 0.87 and ranged from 0.59 to 0.83 for the subscales. 0.83 (95\% CI=0.76-0.90) intraclass correlation coefficient on repeated application of the measure suggested perfect agreement.

Conclusion: Evaluation of the child perceptions questionnaire on oral health related quality of life among school children with various orofacia conditions in Mysore city showed acceptable reliability and construct validity. It also indicated that the impact of the child oral and oro-facial conditions on the functional and psychosocial well-being was significant, and that children were able to give psychometrically acceptable accounts of that impact.

Keywords: Child perceptions, Dental caries, Gingivitis, OHRQoL, Oral impact, Questionnaire.

\section{Introduction}

Traditionally oral health has been measured using clinical data that are mouth- centered and rely on the dental professionals' judgments. The use of clinical indicators alone has been criticized as these give little indication of subjectively perceived symptoms such as pain and discomfort and do not capture the impact of the oral cavity on the person as a whole. The last decade has seen increasing recognition of the value of measures of oral-health-related quality of life in supplementing clinical indicators.

Jokovic et.al reported on the child perceptions questionnaire designed to measure OHRQoL among children aged between 11-14 years. The CPQ for 11-14 year olds is a self -administered questionnaire composed of 37 items enquiring about impacts on four health domains: oral symptoms, functional limitations, emotional well-being and social well-being during the previous three months.

The discriminative properties (i.e., cross- sectional validity and test-retest reliability) of the CPQ are found acceptable in various studies. However, there have been no reports from populations in our country and question remains about the performance of the CPQ in child populations which exhibit the full distribution of clinical presentations.

It is important that the discriminative properties of such measures be acceptable in these populations. Their ability to distinguish between individuals with poor OHRQoL and those with better OHRQoL is a key characteristic which would enable such instruments to contribute to improvements in oral health, through identifying those clinical or public health interventions which produce the greatest improvement in OHRQoL 
Asian Journal of Basic Science \& Research

Volume 2, Issue 3, Pages 23-27, July-September 2020

The method of assessment of validity is based on a model of disease and its consequences proposed by Locker et al 2001.

Clinical status $\longrightarrow$ Symptoms, functional, psychological impact $\longrightarrow$ life overall

This model describes overall quality of life at one end and linear biological, physiological and clinical variables at the other.

A link between symptoms of disease and functional, psychological and social experiences of an individual related to that disease. This study aims to investigate the influence of clinical conditions, and subjective perceptions of school children about oral health on OHRQoL and to assess the reliability and validity of the child perceptions questionnaire.

\section{Methodology}

Before commencing the study, official permission were obtained from the concerned school authorities. 3 schools were randomly selected from by simple random sampling using the table of random numbers for the study. Prevalence of caries was considered for sample size calculation (Jokovic et. al. 2002).

Correlation coefficient of 0.64 at an alpha of 0.01 was found between the number of decayed teeth and the CPQ 11-14 scores (Jokovic et. al. 2002) requiring a total sample of 22 to be significant. So for the present study a sample of 30 children fulfilling the inclusion criteria from each of 3 schools (i.e. 90 in total) was chosen to allow for possible cultural differences.

\section{Inclusion criteria:}

$\checkmark$ School children willing to participate.

$\checkmark$ School children having variety of dental, and oro- facial conditions

$\checkmark$ Informed consent from parents and school authorities

\section{Exclusion criteria:}

$\checkmark$ School children having systemic diseases and under medication.

The school children were asked to complete the child perceptions questionnaire, composed of 37 items enquiring about impacts on four health domains (subscales): oral symptoms, functional limitations, emotional well-being and social well-being

The clinical data on caries status, dental opacities and gingivitis were collected. Descriptive statistical analysis has been carried out in the present study. Cronbach's alpha for the total scale to check the internal consistency and intraclass correlation coefficient were obtained for construct validity. The results were statistically analyzed using the SPSS version 15.0.

\section{Results}

90 children aged 11-14 years formed the sample with the mean age of participants 12.4 years. 
Table-1 Depicts the caries experience of the sample.

\begin{tabular}{|l|l|}
\hline Caries Experience & Mean \pm SD \\
\hline Missing teeth & $0.07 \pm 0.41$ \\
\hline Filled teeth & $0.77 \pm 2.12$ \\
\hline Decayed teeth & $0.38 \pm 1.20$ \\
\hline Decayed, missing, filled teeth & $1.24 \pm 2.75$ \\
\hline
\end{tabular}

The mean number of DMFT was 1.25 (SD 2.75). Untreated decay was present among $15 \%$ of the sample and 35\% had DMFT score of greater than zero.

Table-2 Shows the Mean scores of CPQ for individual subscales

\begin{tabular}{|l|l|}
\hline SUBSCALES & MEAN \pm SD \\
\hline Symptoms & $5.34 \pm 3.26$ \\
\hline Functional & $5.43 \pm 3.85$ \\
\hline Emotional & $4.23 \pm 4.46$ \\
\hline Social & $3.07 \pm 3.09$ \\
\hline
\end{tabular}

Table-3 Depicts Reliability of the total scale and subscales

\begin{tabular}{|l|l|l|l|}
\hline & NO OF ITEMS & CRONBACH'S ALPHA & INTRACLASS CORRELATION \\
\hline Total scale & 37 & 0.87 & 0.89 \\
\hline Symptoms subscale & 6 & 0.63 & 0.76 \\
\hline Functional subscale & 9 & 0.59 & 0.73 \\
\hline Emotional subscale & 9 & 0.83 & 0.85 \\
\hline Social subscale & 13 & 0.65 & 0.78 \\
\hline
\end{tabular}

Substantial to excellent internal consistency was obtained with the Cronbach's alpha ranging from 0.59-0.83 for the sub scales and, for the total scale it was 0.87 (Table 3).

Two weeks later Seventy-nine percent of participants completed the questionnaire for the second time. From the second questionnaires $73 \%(n=43)$ were analysed for test-retest reliability who reported the condition of their mouth was unchanged. 0.83 (95\% $\mathrm{CI}=0.76-0.90)$ intraclass correlation coefficient on repeated application of the measure suggested almost perfect agreement (Landis and Koch, 1977). 
Table-4 Shows the Relationship between clinical data and CPQ scores.

\begin{tabular}{|l|l|l|l|l|l|l|}
\hline Clinical data & Total & $\begin{array}{l}\text { often or } \\
\text { everyday }\end{array}$ & $\begin{array}{l}\text { Symptom } \\
\text { subscale }\end{array}$ & $\begin{array}{l}\text { Functional } \\
\text { subscale }\end{array}$ & $\begin{array}{l}\text { Emotional } \\
\text { subscale }\end{array}$ & $\begin{array}{l}\text { Social } \\
\text { subscale }\end{array}$ \\
\hline Missing teeth & 0.05 & $0.21^{*}$ & 0.08 & 0.11 & 0.11 & 0.01 \\
\hline Filled teeth & 0.09 & 0.10 & -0.01 & 0.16 & 0.11 & 0.06 \\
\hline Decayed teeth & 0.01 & 0.17 & 0.09 & 0.11 & 0.08 & 0.11 \\
\hline DMFT & 0.09 & 0.05 & 0.03 & 0.11 & 0.06 & 0.09 \\
\hline Opacity present & 0.11 & 0.05 & 0.19 & $0.02^{*}$ & 0.53 & 0.14 \\
\hline Gingivitis & 0.54 & 0.47 & 0.72 & 0.31 & 0.82 & 0.99 \\
\hline
\end{tabular}

The measures of CPQ and clinical data is summarized in table-4.The number of children with impacts 'often' or 'everyday' correlated with the total number of missing teeth.

\section{Discussion}

This study aimed to investigate the influence of clinical conditions and subjective perceptions of school children about oral health on OHRQoL and to assess the reliability and validity of the child perceptions questionnaire. Overall these data suggest the CPQ has acceptable reliability with the internal consistency and test-retest reliability. Cronbach's alphas and intraclass correlation coefficient found in this study are equivalent to the results from Canada, UK.

The link between patient-based measures and clinical indicators (Cushing et al.. 1986: Locker and Slade. 1994) in other studies were feeble. These weak relationships between CPQ and clinical data were because of low disease levels in the sample, the conditions under investigation may have caused immeasurably low levels of impact or that the impacts were mediated by a variety of factors such as culture and deprivation.

Only three studies used caries data to measure OHRQoL which was found in review on validation studies. (Jokovic et al., 2002: Robinson et al. 2003; Slade, 1997b). Only fifteen percent of children in this sample had untreated caries compared to 53\% in the Canadian study despite the setting for the evaluation being similar. Mean DMFT levels were similarly lower therefore the degree of impact at these levels of disease on children may be too low for the CPQ to detect.

The CPQ was developed for use in evaluative research for children with oral disease and dental treatment needs. However, the findings from this study suggest that it may not be suitable for discriminating between groups in cross-sectional research in low disease populations.

Finally, the inconsistencies found in the relationship between the clinical data and the CPQ, scores may not be due to the psychometric properties of the measure but due to the fact that impacts are mediated by other factors. It has 
Asian Journal of Basic Science \& Research

been suggested that culture, social and material deprivation can influence the extent of the impact of disease (Locker. 1992)

Variables such as general health status, household income and life stress have been shown to explain as much variance in the impact of oral disorders on adults as clinical indicators such as missing teeth (Locker and Slade, 1994). We have not been able to account for these variables in analyzing the data obtained in the study.

\section{Conclusion}

The CPQ shows good reliability but poor construct validity in relation to clinical variables in this relatively low disease population. If this measure is to be used in similar settings involving children with low levels of caries a large sample size will be required.

\section{References}

1. Jocokic , D Locker, M.Stephens, D.Kenny, B.Tompson, G.Guyatt, Validity and reliability of a questionnaire for measuring child oral - health-related quality of life. J Dent Res, 2002.81 (7): 459-463

2. L.A. Foster Page, W.M.Thomson, A. Jocokic , D Locker, Validation of the child perceptions questionnaire ( CPQ 11-14) J Dent Res 2005,84 (7): 649-652

3. Janice S Paula1, Isabel CG Leite, Anderso B Almeida, Glaucia MB Ambrosano1, Antônio C Pereira1 and Fábio L Mialhe1. The influence of oral health conditions, socioeconomic status and home environment factors on schoolchildren's self-perception of quality of life. Health and Quality of Life Outcomes 2012, 10:6

4. Barbosa TS, Gavião MB: Oral health-related quality of life in children: Part II. Effects of clinical oral health status. A systematic review. Int J Dent Hyg 2008, 6:100-107.

5. Jokovic A, Locker D, Guyatt G: Short forms of the Child Perceptions Questionnaire for 11-14-year-old children (CPQ11-14): Development and initial evaluation. Health Qual Life Outcomes 2006, 4:4.

6. Newton JT, Bower EJ: The social determinants of health: new approaches to conceptualizing and researching complex causal networks. Community Dent Oral Epidemiol 2005, 33:25-34.

7. Biazevic MGH, Rissotto RR, Michel-Crosato E, Mendes LA, Mendes MOA: Relationship between oral health and its impact on quality of life among adolescents. Braz Oral Res 2008, 22(1):36-42.

8. Do LG, Spencer A: Oral Health-Related Quality of Life of children by dental caries and fluorosis experience. J Public Health Dent 2007, 67:132-9.

9. Foster Page LA, Thomson WM, Jokovic A, Locker D: Validation of the Child Perceptions Questionnaire for 11-14 year olds in a population-based study. J Dent Res 2005, 84:649-52.

10.Marshman Z, Rodd H, Stern M, Mitchell C, Locker D, Jokovic A, Robinson PG: An evaluation of the child perceptions questionnaire in the UK. Community Dent Health 2005, 22:151-5

11. Barbosa TS, Tureli MCM, Gavião MBD: Validity and reliability of the Child Perceptions Questionnaires. BMC Oral Health 2009, 9:13.

12.Locker D: Disparities in oral health-related quality of life in a population of Canadian children. Community Dent Oral Epidemiol 2007, 35:348-56. 